\title{
Multiple-Parameter Hamiltonian Approach for Higher Accurate Approximations of a Nonlinear Oscillator with Discontinuity
}

\author{
Najeeb Alam Khan, ${ }^{1}$ Muhammad Jamil, ${ }^{2,3}$ and Asmat Ara ${ }^{1}$ \\ ${ }^{1}$ Department of Mathematics, University of Karachi, Karachi 75270, Pakistan \\ ${ }^{2}$ Abdul Salam School of Mathematical Sciences, GC University, Lahore, Pakistan \\ ${ }^{3}$ Department of Mathematics, NED University of Engineering and Technology, Karachi 75270, Pakistan
}

Correspondence should be addressed to Najeeb Alam Khan, njbalam@yahoo.com

Received 28 May 2011; Accepted 14 June 2011

Academic Editor: Leonid Berezansky

Copyright (C) 2011 Najeeb Alam Khan et al. This is an open access article distributed under the Creative Commons Attribution License, which permits unrestricted use, distribution, and reproduction in any medium, provided the original work is properly cited.

\begin{abstract}
We applied a new approach to obtain natural frequency of the nonlinear oscillator with discontinuity. He's Hamiltonian approach is modified for nonlinear oscillator with discontinuity for which the elastic force term is proportional to $\operatorname{sgn}(u)$. We employed this method for higherorder approximate solution of the nonlinear oscillator equation. This property is used to obtain approximate frequency-amplitude relationship of a nonlinear oscillator with high accuracy. Many numerical results are given to prove the efficiency of the suggested technique.
\end{abstract}

\section{Introduction}

The study of nonlinear oscillator problems is of crucial importance not only in all areas of physics but also in engineering and other disciplines. It is of great importance to study analytically nonlinear oscillators to obtain approximate frequency-amplitude relationship because of their wide applications. Traditional perturbation method provides us with a simple approach to the determination of the frequency-amplitude relationship, but the results are valid only for special cases, that is, for weakly nonlinear systems or for the case when the amplitude is very small. In order to overcome the shortcomings arising in traditional perturbation methods, various alternative approaches have been proposed, for example, variational iteration method [1-3], homotopy perturbation method [4-7], Lindstedt-Poincare method [8], variational approach [9, 10], parameter-expanding method [11] and max-min approach [12], harmonic balance method [13], and Hamiltonian approach [14]. 
In the present study, the mentioned parameters are those undetermined values in the assumed solution. In the three-parameters technique, the motion is assumed as $u=$ $A_{0} \cos \omega t+A_{1} \cos 3 \omega t$, where $\omega, A_{0}, A_{1}$ are the angular frequency of motion and Fourier coefficients, respectively. The three undetermined parameters are determined by using the governing equation of motion and the initial conditions imposed. The way for obtaining the parameters in He's Hamiltonian technique is quite different from that in the harmonic balance method. Therefore, the present technique is not the same as the harmonic balance method. Finally, the paper provides a lot of higher accurate results for the angular frequency $\omega$ of the motion.

\section{Analysis}

In this paper, we consider a general form of nonlinear oscillator

$$
\ddot{u}+f(u)=0
$$

with initial conditions $u(0)=A$ and $\dot{u}(0)=0$.

The variational principle for (2.1) suggested by He [9] can be written as

$$
J(u)=\int_{0}^{T / 4}\left(\frac{\dot{u}^{2}}{2}-F(u)\right) d t
$$

where $T$ is period of the nonlinear oscillator, $\partial F / \partial u=f$. In the functional $(2.2), \dot{u}^{2} / 2$ is the kinetic energy, so that the functional (2.2) is the least Lagrangian action, from which we can write the Hamiltonian

$$
H=\frac{\dot{u}^{2}}{2}+F(u)=\text { constant. }
$$

In previous work [14], the trail function $u=A \cos w t$. In this paper, we consider a general trail function is of the form $u=\sum_{i=0}^{n} A_{i} \cos ((2 i+1) \omega t)$. From (2.3), we have

$$
\frac{\partial H}{\partial A_{i}}=0
$$

Introducing a new function, $\widehat{H}(u)$ defined as

$$
\widehat{H}(u)=\int_{0}^{T / 4}\left(\frac{\dot{u}^{2}}{2}+F(u)\right) d t=\frac{1}{4} T H .
$$

It is obvious that

$$
\frac{\partial \widehat{H}}{\partial T}=\frac{H}{4}
$$


Equation (2.4) is, then, equivalent to the following one

$$
\frac{\partial}{\partial A_{i}}\left(\frac{\partial \widehat{H}}{\partial T}\right)=0
$$

or

$$
\frac{\partial}{\partial A_{i}}\left(\frac{\partial \widehat{H}}{\partial(1 / w)}\right)=0, \quad i=0,1,2, \ldots, n
$$

From system of (2.7a) and (2.7b), we can obtain approximate frequency-amplitude relationship of a nonlinear oscillator up to higher order.

\section{Approximation by Multiple-Parameter Hamiltonian Technique}

Consider the following nonlinear oscillator with discontinuity

$$
\ddot{u}+\operatorname{sgn}(u)=0
$$

with initial conditions $u(0)=A$ and $\dot{u}(0)=0$.

Here, we suppose that $f(u)=\operatorname{sgn}(u)$ such that

$$
\operatorname{sgn}(u)= \begin{cases}-1, & u<0 \\ 1, & u \geq 0\end{cases}
$$

Therefore,

$$
f(u)= \begin{cases}-1, & u<0 \\ 1, & u>0\end{cases}
$$

Its variational formulation can be written as

$$
J(u)=\int_{0}^{T / 4}\left(-\frac{\dot{u}^{2}}{2}+u\right) d t+\int_{T / 4}^{T / 2}\left(-\frac{\dot{u}^{2}}{2}-u\right) d t
$$

and $\widehat{H}(u)$ can be written in the form as

$$
\widehat{H}(u)=\int_{0}^{T / 4}\left(\frac{\dot{u}^{2}}{2}+u\right) d t+\int_{T / 4}^{T / 2}\left(\frac{\dot{u}^{2}}{2}-u\right) d t .
$$

Using $u=\sum_{i=0}^{n} A_{i} \cos ((2 i+1) \omega t)$ as an approximate solution. Here, we consider second-, third-, and higher-order Hamiltonian approach. The Hamiltonian approach applied by He [14] and obtained the frequency-amplitude relationship $\omega_{1 \text { (app) }}=1.128379 / \sqrt{A}$ for the firstorder approximation of $u=A_{0} \cos \omega t$. 
To obtain more accurate results assume that the solution can be expressed as

$$
u=A_{0} \cos \omega t+A_{1} \cos 3 \omega t
$$

According to the initial conditions:

$$
A=A_{0}+A_{1}
$$

Inserting (3.6) in (3.5), we obtain

$$
\begin{aligned}
\widehat{H}(u)= & \int_{0}^{T / 4}\left(\frac{1}{2}\left(A_{0} \omega \sin \omega t+3 A_{1} \omega \sin 3 \omega t\right)^{2}+\left(A_{0} \cos \omega t+A_{1} \cos 3 \omega t\right)\right) d t \\
+ & \int_{T / 4}^{T / 2}\left(\frac{1}{2}\left(A_{0} \omega \sin \omega t+3 A_{1} \omega \sin 3 \omega t\right)^{2}-\left(A_{0} \cos \omega t+A_{1} \cos 3 \omega t\right)\right) d t \\
\widehat{H}(u)= & \int_{0}^{\pi / 2}\left(\frac{\omega}{2}\left(A_{0} \sin t+3 A_{1} \sin 3 t\right)^{2}+\frac{1}{\omega}\left(A_{0} \cos t+A_{1} \cos 3 t\right)\right) d t \\
& +\int_{\pi / 2}^{\pi}\left(\frac{\omega}{2}\left(A_{0} \sin t+3 A_{1} \sin 3 t\right)^{2}-\frac{1}{\omega}\left(A_{0} \cos t+A_{1} \cos 3 t\right)\right) d t \\
\widehat{H}(u)= & \frac{6 A_{0}-2 A_{1}}{3 \omega}+\frac{\pi \omega\left(A_{0}^{2}+9 A_{1}^{2}\right)}{4}
\end{aligned}
$$

setting

$$
\begin{gathered}
\frac{\partial}{\partial A_{0}}\left(\frac{\partial \widehat{H}}{\partial(1 / \omega)}\right)=2-\frac{\omega^{2} \pi A_{0}}{2}=0 \\
\frac{\partial}{\partial A_{1}}\left(\frac{\partial \widehat{H}}{\partial(1 / \omega)}\right)=-\frac{2}{3}-\frac{9 \omega^{2} \pi A_{1}}{2}=0 .
\end{gathered}
$$

After some mathematical simplification by using Mathematica built-in utilities, we achieved

$$
A_{0}=1.038461 A, \quad A_{1}=-0.038461 A \text {. }
$$

We obtained the following frequency-amplitude relationship for nonlinear oscillator with discontinuity:

$$
\omega_{2(\mathrm{app})}=\frac{1.107286}{\sqrt{A}} .
$$


Since the accuracy of the obtained results in three-parameters technique is not so high, the four parameters technique is introduced as follows:

$$
u=A_{0} \cos \omega t+A_{1} \cos 3 \omega t+A_{2} \cos 5 \omega t
$$

where $A_{0}, A_{1}, A_{2}, \omega$ are four undetermined parameters. According to the initial conditions:

$$
\begin{gathered}
A=A_{0}+A_{1}+A_{2}, \\
\frac{\partial}{\partial A_{0}}\left(\frac{\partial \widehat{H}}{\partial(1 / \omega)}\right)=2-\frac{\omega^{2} \pi A_{0}}{2}=0, \\
\frac{\partial}{\partial A_{1}}\left(\frac{\partial \widehat{H}}{\partial(1 / \omega)}\right)=-\frac{2}{3}-\frac{9 \omega^{2} \pi A_{1}}{2}=0, \\
\frac{\partial}{\partial A_{2}}\left(\frac{\partial \widehat{H}}{\partial(1 / \omega)}\right)=\frac{2}{5}-\frac{25 \omega^{2} \pi A_{2}}{2}=0 .
\end{gathered}
$$

After some mathematical simplification by using Mathematica built-in utilities, we achieved

$$
A_{0}=1.029905 A, \quad A_{1}=-0.038144 A, \quad A_{2}=0.008239 A \text {. }
$$

We obtained the following frequency-amplitude relationship for nonlinear oscillator with discontinuity

$$
\omega_{3(\mathrm{app})}=\frac{1.111876}{\sqrt{A}}
$$

which is very close to the exact one $[1,14,15]$

$$
\omega_{\text {Exact }}=\frac{\pi}{2 \sqrt{2 A}}=\frac{1.110721}{\sqrt{A}} .
$$

\section{Discussion and Conclusion}

The present method is an extremely simple method, leading to high accuracy of the obtained results. The main merit of the method is that the obtained results are valid for the whole solution domain. In this study, we obtained the relative error as $0.309 \%$ for the secondorder approximation while the other researchers $[1,8]$ obtained the relative error as $1.8 \%$. The reason for the difference in the relative error is that the other researchers take less precision in the decimal numbers during calculations. We reached $0.103 \%, 0.046 \%, 0.024 \%$, $0.014 \%, 0.009 \%$, and $0.006 \%$ relative errors for the third, fourth, fifth, sixth, seventh, and eighth approximate periods, respectively. However, the calculations of the higher-order approximation are not presented. One can obtain higher-order accuracy by extending the idea given in this paper. Comparison of the multiple-parameter Hamiltonian solution with numerical solution obtained by Mathematica built-in utilities is given in Figure 1. 


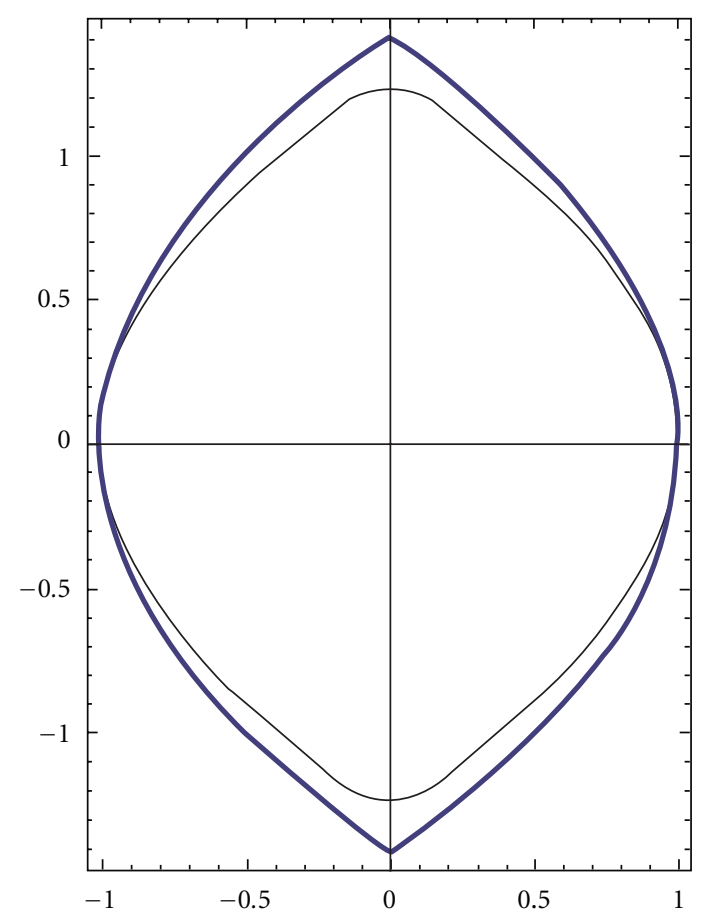

(a)

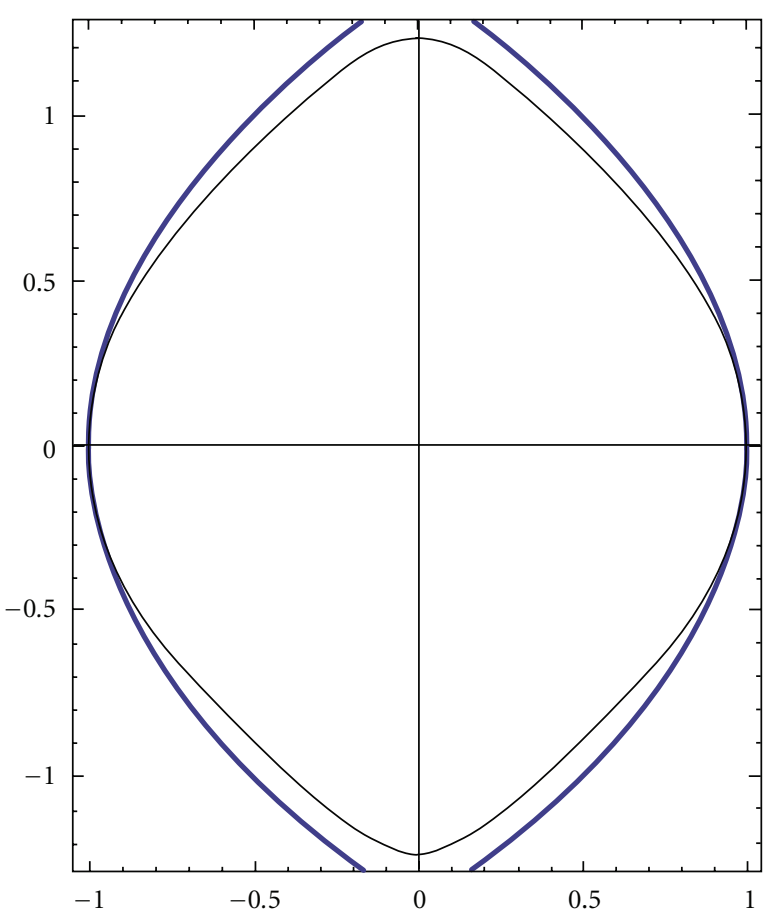

(b)

Figure 1: Comparison for the $u$ versus $\dot{u}$ trajectory for the case of $A=1$; bold line represents the numerical solution; and black line represents the multiple-parameter Hamiltonian approach (a) six-parameter (b) eight-parameter. 


\section{Acknowledgments}

The author N. A. Khan is highly thankful and grateful to the Dean of Faculty of Sciences, University of Karachi, Karachi-75270, Pakistan for supporting and facilitating this research work. The author M. Jamil is highly thankful and grateful to the Abdus Salam School of Mathematical Sciences, GC University, Lahore, Pakistan, the Department of Mathematics, NED University of Engineering \& Technology, Karachi-75270, Pakistan, and also the Higher Education Commission of Pakistan for generous support and facilitating this research work.

\section{References}

[1] M. Rafei, D. D. Ganji, H. Daniali, and H. Pashaei, "The variational iteration method for nonlinear oscillators with discontinuities," Journal of Sound and Vibration, vol. 305, no. 4-5, pp. 614-620, 2007.

[2] L.-N. Zhang and J.-H. He, "Resonance in Sirospun yarn spinning using a variational iteration method," Computers \& Mathematics with Applications, vol. 54, no. 7-8, pp. 1064-1066, 2007.

[3] T. Öziş and A. Yıldırım, "A study of nonlinear oscillators with $\mathrm{u}^{1 / 3}$ force by He's variational iteration method," Journal of Sound and Vibration, vol. 306, no. 1-2, pp. 372-376, 2007.

[4] A. Beléndez, C. Pascual, M. Ortuño, T. Beléndez, and S. Gallego, "Application of a modified He’s homotopy perturbation method to obtain higher-order approximations to a nonlinear oscillator with discontinuities," Nonlinear Analysis: Real World Applications, vol. 10, no. 2, pp. 601-610, 2009.

[5] A. Beléndez, C. Pascual, S. Gallego, M. Ortuño, and C. Neipp, "Application of a modified He's homotopy perturbation method to obtain higher-order approximations of an $x^{1 / 3}$ force nonlinear oscillator," Physics Letters A, vol. 371, no. 5-6, pp. 421-426, 2007.

[6] J.-H. He, "The homotopy perturbation method nonlinear oscillators with discontinuities," Applied Mathematics and Computation, vol. 151, no. 1, pp. 287-292, 2004.

[7] A. Beléndez, A. Hernández, T. Beléndez, E. Fernández, M. L. Álvarez, and C. Neipp, “Application of He's homotopy perturbation method to the Duffin-harmonic oscillator," International Journal of Nonlinear Sciences and Numerical Simulation, vol. 8, no. 1, pp. 79-88, 2007.

[8] H.-M. Liu, "Approximate period of nonlinear oscillators with discontinuities by modified LindstedtPoincare method," Chaos, Solitons and Fractals, vol. 23, no. 2, pp. 577-579, 2005.

[9] J.-H. He, "Variational approach for nonlinear oscillators," Chaos, Solitons and Fractals, vol. 34, no. 5, pp. 1430-1439, 2007.

[10] D.-H. Shou, "Variational approach to the nonlinear oscillator of a mass attached to a stretched wire," Physica Scripta, vol. 77, no. 4, Article ID 045006, 2008.

[11] F. Ö. Zengin, M. O. Kaya, and S. A. Demirbă̆, "Application of parameter-expansion method to nonlinear oscillators with discontinuities," International Journal of Nonlinear Sciences and Numerical Simulation, vol. 9, no. 3, pp. 267-270, 2008.

[12] J.-H. He, "Max-min approach to nonlinear oscillators," International Journal of Nonlinear Sciences and Numerical Simulation, vol. 9, no. 2, pp. 207-210, 2008.

[13] Z. Guo and A. Y. T. Leung, "The iterative homotopy harmonic balance method for conservative Helmholtz-Duffing oscillators," Applied Mathematics and Computation, vol. 215, no. 9, pp. 3163-3169, 2010.

[14] J.-H. He, “Hamiltonian approach to nonlinear oscillators," Physics Letters. A, vol. 374, no. 23, pp. 23122314, 2010.

[15] Z.-F. Ren and J.-H. He, “A simple approach to nonlinear oscillators," Physics Letters. A, vol. 373, no. 41, pp. 3749-3752, 2009. 


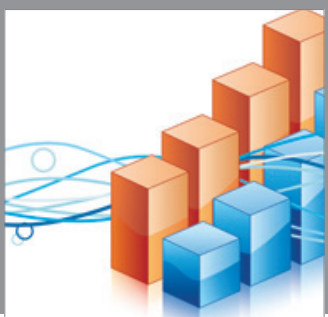

Advances in

Operations Research

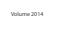

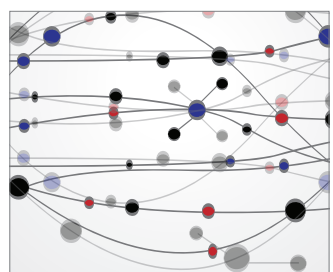

\section{The Scientific} World Journal
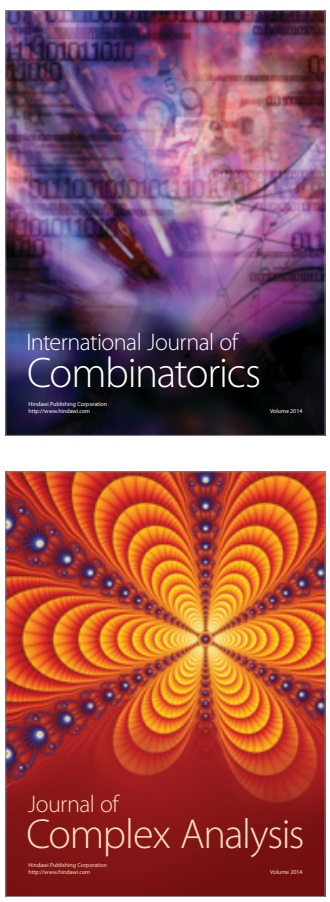

International Journal of

Mathematics and

Mathematical

Sciences
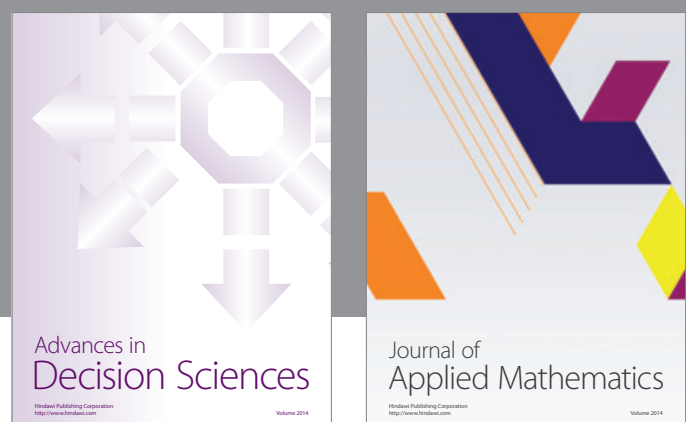

Journal of

Applied Mathematics
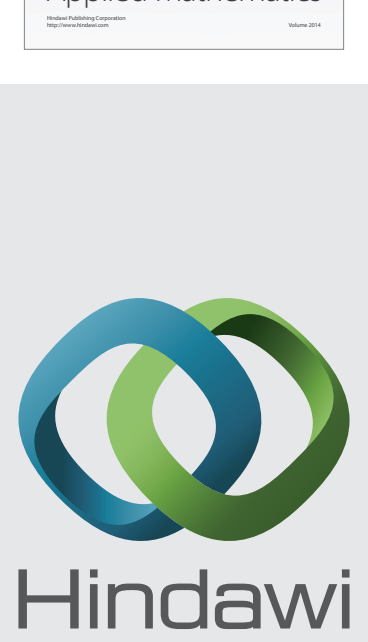

Submit your manuscripts at http://www.hindawi.com
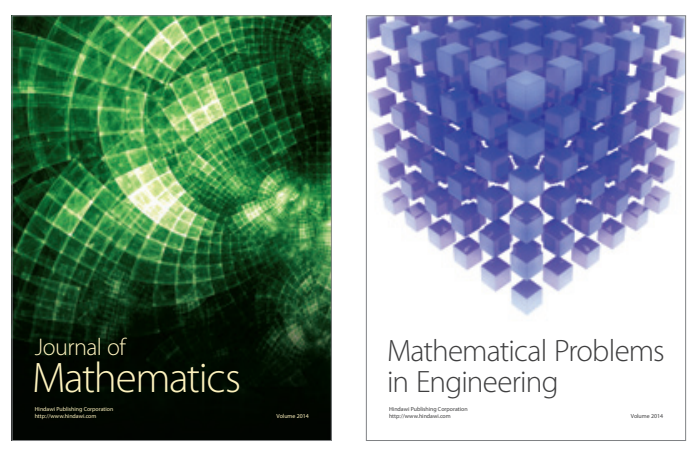

Mathematical Problems in Engineering
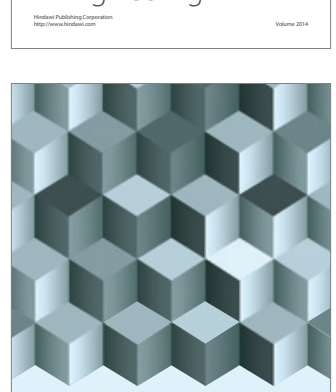

Journal of

Function Spaces
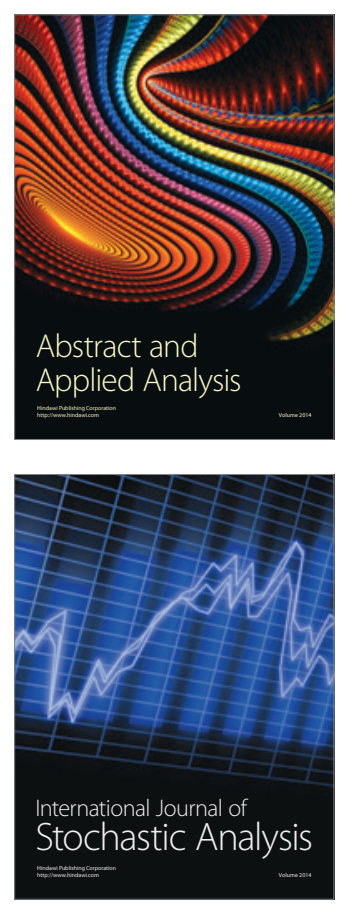

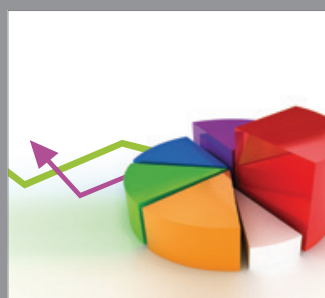

ournal of

Probability and Statistics

Promensencen
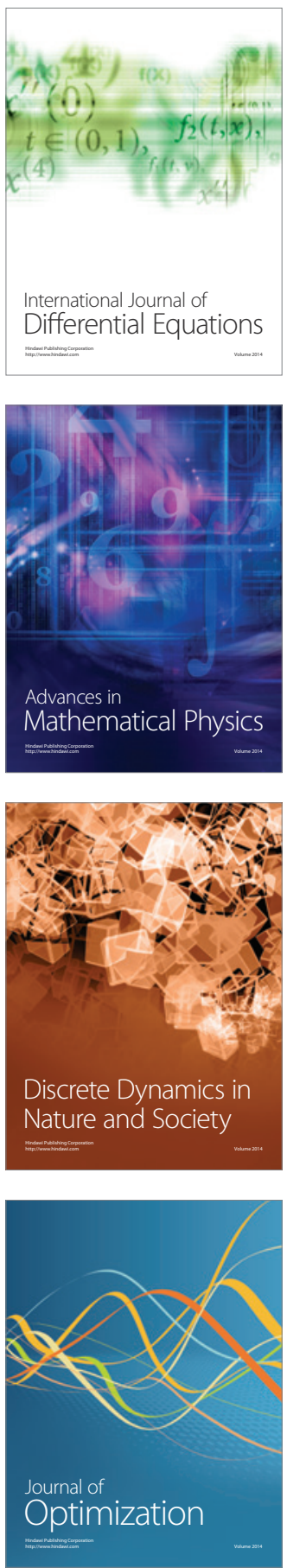